This item was submitted to Loughborough's Research Repository by the author.

Items in Figshare are protected by copyright, with all rights reserved, unless otherwise indicated.

\title{
Aspect ratio distribution and chord length distribution driven modeling of crystallization of two-dimensional crystals for real-time model-based applications
}

\section{PLEASE CITE THE PUBLISHED VERSION}

https://doi.org/10.1021/acs.cgd.8b00758

\section{PUBLISHER}

(c) American Chemical Society

\section{VERSION}

AM (Accepted Manuscript)

\section{PUBLISHER STATEMENT}

This work is made available according to the conditions of the Creative Commons Attribution-NonCommercialNoDerivatives 4.0 International (CC BY-NC-ND 4.0) licence. Full details of this licence are available at: https://creativecommons.org/licenses/by-nc-nd/4.0/

\section{LICENCE}

CC BY-NC-ND 4.0

\section{REPOSITORY RECORD}

Szilagyi, Botond, and Zoltan K. Nagy. 2018. "Aspect Ratio Distribution and Chord Length Distribution Driven Modeling of Crystallization of Two-dimensional Crystals for Real-time Model-based Applications". figshare. https://hdl.handle.net/2134/36082. 


\title{
Aspect ratio distribution and chord length
}

\section{distribution driven modeling of crystallization of}

\section{two dimensional crystals for real-time model based}

\section{applications}

\author{
Botond Szilágyi ${ }^{\dagger}$, Zoltán K. Nagy ${ }^{*} \dagger^{*}$
}

${ }^{\dagger}$ Davidson School of Chemical Engineering, Purdue University, West Lafayette, IN 47907-2100, USA

Department of Chemical Engineering, Loughborough University, Loughborough, Leicestershire, LE11 3TU, United Kingdom

Keywords: population balance models, crystallization, GPU acceleration, soft-sensors, artificial neural network

\begin{abstract}
Two dimensional crystals, for which the shape is described by two linear sizes are common in fine chemical and pharmaceutical industries. Since the crystal size and shape are directly related to the performance of active pharmaceutical ingredients (API), the simultaneous size and shape
\end{abstract}


distribution control is of paramount importance in pharmaceutical crystallization engineering. To efficiently achieve simultaneous size and shape control often requires model based control strategies, however the increased computational cost of the process simulation and the substantial differences between the simulated and measurable quantities make the implementation of model based control approaches challenging. This paper addresses the important problem of the real-time simulation of the most likely measurable chord length distribution (CLD) and aspect ratio distribution (ARD) as well as the concentration variations during the crystallization of 2D needleshape crystals. This enables the application of focused beam reflectance measurement (FBRM) and particle vision and microscopy (PVM), two routinely applied probes, as quantitative direct feedback control tools. Artificial neural network (ANN) based FBRM and PVM soft-sensors are developed, which enable the direct and fast transformation of 2D crystal size distribution (CSD) to CLD and ARD on arbitrary 2D grids. The training data for the ANN is generated by a first principle, geometrical model based simulation of FBRM and PVM for high aspect ratio crystals. Although, the ANN approach is applicable for any simulated or experimental training datasets. It is also demonstrated that the in-situ imaging based shape measurement underestimates the real aspect ratio (AR) of crystals, for which a simple correction is proposed. From the model-equation solution perspective, the soft-sensors require full $\mathrm{PB}$ solution. The 2D high resolution finite volume method (HR-FVM) is applied to simulate the full 2D CSD, which is an accurate, stable, but computationally expensive technique. The real time applicability is achieved through various implementation improvements including grid optimization and data-type optimized hybrid CPUGPU calculations.

\section{Introduction}


The crystal size distribution (CSD) of particulate materials has a major impact on downstream operations (filtration, drying, granulation, milling, storage) and it can be crucial in the pharmaceutical industry as it influences many properties of the active pharmaceutical ingredients (APIs), including dissolution rate and bioavailability. It has been shown that the vast majority of APIs have a crystal length-to-width ratio, also called aspect ratio (AR), larger than one, having elongated or often needle-like crystal shape. ${ }^{1,2}$ The crystallization behavior of these high AR crystals were profoundly investigated ${ }^{3-5}$ motivated by the fact that crystal shape has been correlated with numerous API properties from sticking propensity ${ }^{6}$ through compressing and densification mechanisms ${ }^{7}$ to bioavailability. ${ }^{8}$

The aforementioned observations have led to the need of simultaneous crystal size and shape control during the crystallization process. ${ }^{9}$ Numerous techniques have been presented, mainly for analyzing ${ }^{10}$ but also for controlling the AR, through supersaturation control (SSC). ${ }^{11}$ It has been demonstrated that varying the supersaturation alone has some, but limited impact on the achievable crystal shape. Contrary, growth rate modifiers are able to generate significant shape variations. ${ }^{12,13}$ Numerous growth rate inhibition mechanisms have been proposed based on empirical observations. Recently molecular dynamic simulations have also been employed for deeper understanding of the underlying mechanisms. ${ }^{14}$ As an alternative of additives, the effects of ultrasound on crystallization process and shape evolution have also been studied. ${ }^{15-17}$

A major barrier to simultaneous size-shape distribution control implementation is the difficulty of in-situ and real time multidimensional CSD ( $n D$ CSD) measurement. Stereoscopic imaging setups were proposed to gather high accuracy $n D$ CSD information. ${ }^{18,19}$ These setups are still in research and development stage, and are not always able to provide real-time information. Focused beam reflectance measurement (FBRM) and particle vision and microscopy (PVM) data are 
routinely collected during crystallization, which provide size and shape related information, but the deconvolution of $n D$ CSD from these signals is rarely possible.

The deconvolution techniques and their applicability of predicting the CSD from measured chord length distribution (CLD) and AR are still intensively investigated. ${ }^{20-24}$ These studies have demonstrated that the back-transformation of CSD from CLD leads to ill-posed optimization problems. The idea of forward-transformation of CLD from CSD was also analyzed: geometrical models, which considered the shape of crystal only, or also included optical models were developed..$^{25,26}$ The general conclusion of these studies is that the forward transformation cannot be generalized reliably for accurate off-line system analysis. However, it is known that even approximate models, in combination with state estimators, can be successfully applied in nonlinear model predictive control (NMPC) ${ }^{27}$ Consequently, there is still need for an approximate, fast transformation, which may not be appropriate for detailed measurement-based system analysis, but given the feedback-based error estimation and correction, it can provide satisfactory information for a model based control system. ${ }^{28}$

Numerous studies deal with crystal shape simulation, ${ }^{29-31}$ which most often involve moments based PBE solution methods. Even though moment based solutions are computationally very efficient, they have the common limitation of not being able to provide the full CSD, only its moments. Full PBE solution is required to enable the use of distributional information provided by the FBRM CLD and aspect ratio distribution (ARD) from image analysis. An accurate but computationally expensive technique, often used for the solution of PBEs is the high resolution finite volume method (HR-FVM). ${ }^{32}$ Advanced implementations of HR-FVM, including parallelization on CPU clusters, ${ }^{33}$ coordinate system transformation ${ }^{34}$ and hybrid parallel CPU- 
GPU programming have enabled remarkable speedup, and brought the real-time solution of PBEs in the realm of possibility. ${ }^{35}$

The objective of this work is the development of a process model for crystallization of 2D rodlike crystals, which also includes sensor models for FBRM and PVM, using a novel fast ANN based 2D CSD to CLD and ARD transformation. The application is designed for real-time control applications, hence, the simulation time reduction is in the focus of model development. The existence of an offset between the real and in-situ imaging based AR is also demonstrated and a shape-dependent correction is proposed.

\section{Crystallization process modeling and simulation}

\section{The $2 D$ population balance model}

This work considers high aspect-ratio crystals, for which the shape is described by two linear size variables: the length $\left(L_{1}\right)$ is considerably longer than the width and height, which are considered to be equal and referred to as width $\left(L_{2}\right)$. Thus, each crystal is described by the $\left(L_{1}, L_{2}\right)$ pair. For the sake of simplicity and better readability the $\left(L_{1}, L_{2}\right) \rightarrow \mathbf{L}$ vector notations will be used wherever is possible. The crystals population is characterized by the bivariate density function $n(\mathbf{L}, t) d \mathbf{L}$, which gives the number of crystals within the $\left(L_{1}, L_{1}+d L_{1}\right) \times\left(L_{2}, L_{2}+d L_{2}\right)$ size domain at $t$ time moment in unit volume of suspension. Considering perfect mixing and homogeneous temperature field, and assuming secondary nucleation and crystal growth mechanisms, the PBE governing 2D CSD dynamics takes the form:

$\frac{\partial n(\mathbf{L}, t)}{\partial t}+\frac{\partial\left[G_{1} n(\mathbf{L}, t)\right]}{\partial L_{1}}+\frac{\partial\left[G_{2} n(\mathbf{L}, t)\right]}{\partial L_{2}}=B \delta\left(\mathbf{L}-\mathbf{L}_{\boldsymbol{n}}\right)$

with the boundary and initial conditions: 
$\lim _{\mathbf{L} \rightarrow \infty} n(\mathbf{L}, t)=0$

$n(\mathbf{L}, t=0)=n_{0}(\mathbf{L})$

In the PB Eq.(1) the first term is the temporal evolution of the bivariate size density function, whereas the second and third terms take into account the growth along the length and width axes. The right hand side is for the nucleation, where $\mathbf{L}_{\boldsymbol{n}}$ denotes the nucleus size.

Secondary nucleation mechanism is considered, described by the power-law formula:

$B=k_{b} \sigma^{b} V_{c}$

where $V_{c}$ stands for the crystals volume fraction, $k_{b}$ and $b$ are material and system specific constants and $\sigma$ is the relative supersaturation:

$$
\sigma=\frac{c-c_{s}}{c_{s}}=\frac{c}{c_{s}}-1
$$

where $c$ denotes the solute concentration and $c_{S}$ is the solubility. The temperature dependency of solubility, expressed in mass fraction, is described by the power law equation:

$$
c_{s}=a_{0}+a_{1} T+a_{2} T^{2}
$$

In the PB Eq. (1) $G_{1}$ and $G_{2}$ are the growth rates along the length and width axes, respectively, which are calculated using the power-law rate equations:

$$
G_{i}=k_{g, i} \sigma^{g i}, i=\{1,2\}
$$

The symbols $k_{g, i}$ and $g i$ are system and material specific constants.

Assuming rod-like crystals, the component balance for the crystallizing material is written as:

$$
\frac{d c}{d t}=-\frac{k_{V} \rho_{c}}{1-V_{c}}\left[\int_{0}^{\infty} G_{1} L_{2}^{2} n(\mathbf{L}, t) d \mathbf{L}+2 \int_{0}^{\infty} G_{2} L_{1} L_{2} n(\mathbf{L}, t) d \mathbf{L}+L_{n}^{3} B\right]
$$

where $\rho_{c}$ is the crystal density and $k_{V}$ is the volumetric shape factor, a transformation constant between the size and volume of a single crystal $\left(v_{c}\right)$ : 


$$
v_{c}=k_{V} L_{1} L_{2}^{2}
$$

The temperature is considered a programmed variable, thus energy balance is not needed in the model. The set of partial and ordinary differential Eqs. (1)-(8) constitutes the mathematical model of the cooling batch crystallization of high AR crystals with secondary nucleation and crystal growth. Analytical solution for this equation system does not exist. The difficulty in numerical solution is that the two dimensional PB Eq.(1) is coupled with the mass balance Eq.(7) and the PBE is a hyperbolic partial differential equation (PDE). The HR-FVM is a generic method for the solution of hyperbolic PDEs, which was also successfully applied for the higher dimensional population balances. ${ }^{32}$ As model system potassium dihydrogen phosphate in water is used, for which the material properties are listed in Table 1 , adapted from the literature. ${ }^{36}$

Table 1. Material and kinetic related model parameters

\begin{tabular}{clll}
\hline Name & Meaning & Value & UM \\
\hline$\rho_{c}$ & crystal density & 2388 & $\mathrm{~kg} / \mathrm{m}^{3}$ \\
$k_{V}$ & shape factor & 1 & - \\
$a_{0}$ & zero order solubility constant & 0.2088 & - \\
$a_{1}$ & first order solubility constant & $-9.76 \cdot 10^{-5}$ & ${ }^{\circ} \mathrm{C}^{-1}$ \\
$a_{2}$ & second order solubility constant & $9.30 \cdot 10^{-5}$ & ${ }^{\circ} \mathrm{C}^{-2}$ \\
$k_{b}$ & nucleation rate constant & $7.49 \cdot 10^{18}$ & ${ }^{3} / \mathrm{m}^{3} \mathrm{~s}$ \\
$b$ & nucleation supersaturation exponent & 2.04 & - \\
$k_{g, 1}$ & length growth rate constant & 100.75 & $\mu \mathrm{m} / \mathrm{s}$ \\
$g_{1}$ & length growth supersaturation exponent & 1.74 & - \\
$k_{g, 2}$ & width growth rate constant & 12.1 & $\mu \mathrm{m} / \mathrm{s}$
\end{tabular}


In this work the CrySiV Matlab based function was used to solve the model equations. ${ }^{35}$

\section{Process simulation and simulation time improvement}

In the simulations a seeded crystallization process is considered, where the seed CSD is given by the un-correlated bivariate log-normal probability density function:

$$
n_{0}\left(L_{1}, L_{2}\right)=\frac{1}{\sqrt{2 \pi} L_{1} L_{2} \sigma_{1} \sigma_{2}} \exp \left[-\sum_{i=1}^{2} \frac{\left(\ln \left(L_{i}\right)-\mu_{i}\right)^{2}}{2 \sigma_{i}}\right]
$$

where:

$$
\begin{gathered}
\mu_{i}=\ln \left(\frac{m_{i}}{\sqrt{1+\frac{v_{i}}{m_{i}^{2}}}}\right) \\
\sigma_{i}=\sqrt{\ln \left(1+\frac{v_{i}}{m_{i}^{2}}\right)}
\end{gathered}
$$

where $m$ denotes the mean, whereas $v$ stands for the variance of the distribution. The seed and process related data are listed in Table 2.

Table 2. Seed and process related data used in the simulations.

\begin{tabular}{clcc}
\hline Name & Meaning & Value & UM \\
\hline$m_{1}$ & mean length of seeds & 200 & $\mu \mathrm{m}$ \\
$v_{1}$ & dispersion around $m_{1}$ & 250 & $\mu \mathrm{m}$ \\
$m_{2}$ & mean width of seeds & 100 & $\mu \mathrm{m}$ \\
$v_{2}$ & dispersion around $m_{2}$ & 150 & $\mu \mathrm{m}$
\end{tabular}




\begin{tabular}{clcc}
$V_{s}$ & seed volume fraction & 0.01 & - \\
$T_{\text {in }}$ & initial temperature & 60 & ${ }^{\circ} \mathrm{C}$ \\
$T_{f}$ & final temperature & 20 & ${ }^{\circ} \mathrm{C}$ \\
$t_{b}$ & batch time & 7200 & $\mathrm{~s}$ \\
\hline
\end{tabular}

The discretization of the 2D PBM using the 2D HR-FVM results in a large number of equations for which the solution requires high computation cost. Thus, the simulation time needs to be improved to meet the ambitious objective of real-time model based control. In this work a series of numerical optimizations are carried out to achieve a computationally highly efficient real-time 2D HR-FVM implementation, including: (i) use of fast programming language (e.g. C, Fortran), (ii) parallelization on graphical processing unit (GPU), (iii) grid optimization, and (iv) data type optimization.

The crystallization process model is solved by the HR-FVM as a compiled C .mex file by the serial $\mathrm{CrySiV}$ solver. The $1000 \times 1000 \mu \mathrm{m}$ grid, based on the kinetic equations, covers the attainable crystal size domain and the $\mathrm{h}=1 \mu \mathrm{m}$ cell size keeps the solution accurate. Figure 1 presents the simulation results. The run time of this simulation is considered the "base case". 


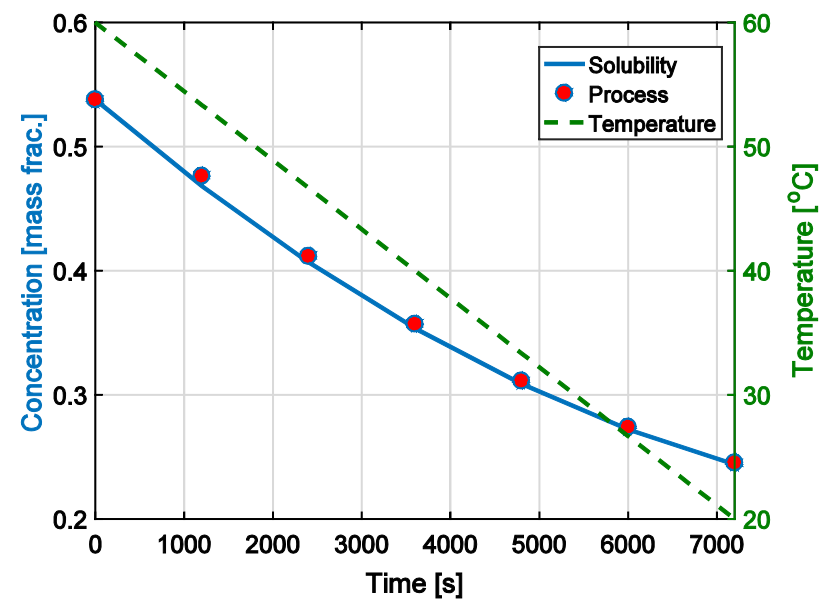

a)
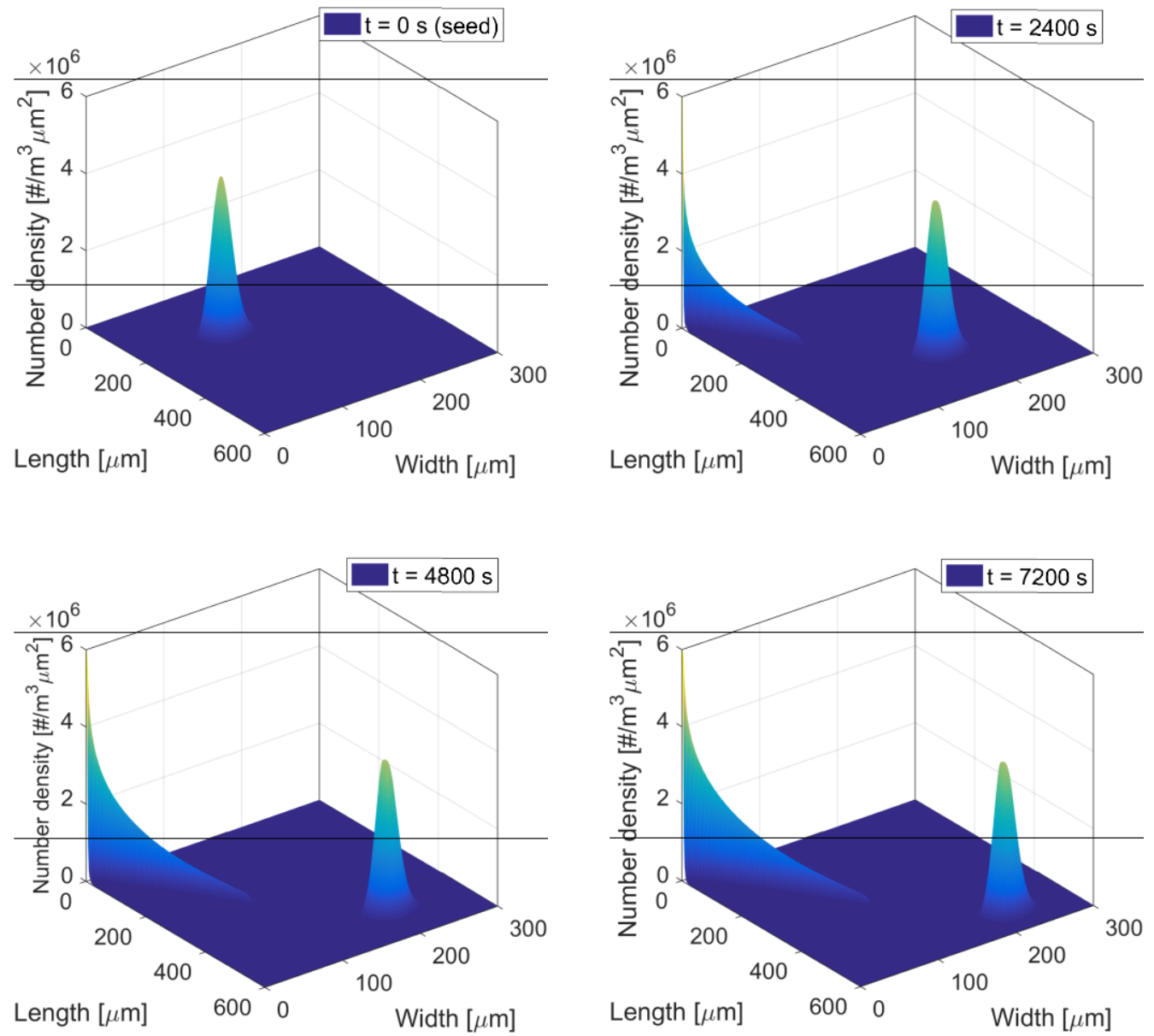

b) 
Figure 1. a) The applied temperature profile with the corresponding solubility and simulated concentrations; b) crystal size distribution in four representative time moments.

The simulation of $7200 \mathrm{~s}$ process time took $132.2 \mathrm{~s}$ (see the machine specifications in Appendix 1), which is within real time, but is not feasible for real-time optimization. Moreover, the sampling time, which translates to available calculation time, is evidently shorter than the full process time.

The 2D $1000 \times 1000 \mu \mathrm{m}$ grid generates $10^{6}$ equations, which are solved in every time step. Due to their massively parallel hardware architecture (see Figure 2), GPUs have been used for the acceleration of various scientific calculations. In this case for optimal CPU-GPU utilization, a hybrid HR-FVM implementation is proposed, according to which the serial operations i.e. calculation of nucleation and growth rates, temperature, mass balance and time stepping are executed in the CPU, whereas the parallelizable HR-FVM and integral calculations are passed to the GPU.

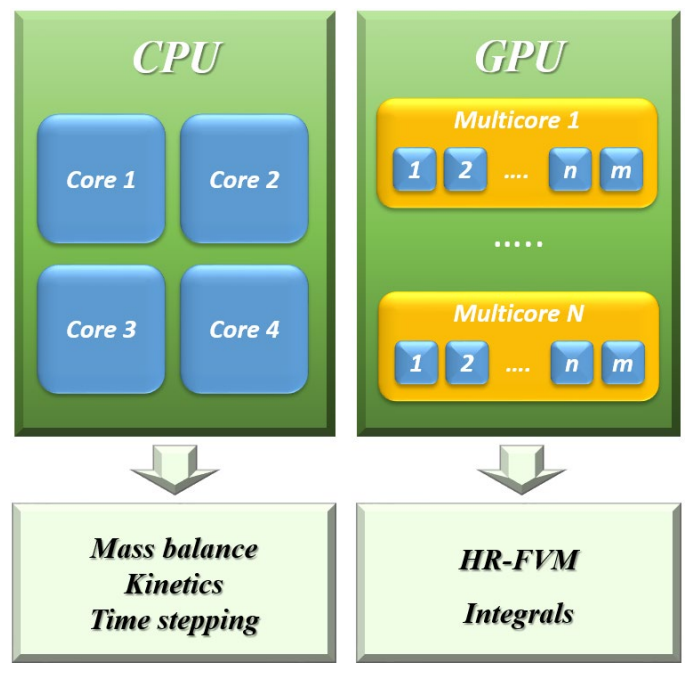

Figure 2. Schematic comparison of CPU and GPU architecture and the assignment of calculations in the hybrid CPU-GPU based 2D HR-FVM implementation. 
The hybrid CPU-GPU solution is carried out using the GPU accelerated CrySiV Matlab function, resulting in a solution time of $11.43 \mathrm{~s}$. Comparing this to the $132.20 \mathrm{~s}$ pure serial solution time it can be seen that the CPU alone cannot over perform the hybrid CPU-GPU performance. Even in the theoretically optimal case, when the four cores would reduce the calculation time to its quarter, the best CPU time would be around $\sim 33.05 \mathrm{~s}$, which is still larger than the computational time achieved with the hybrid CPU-GPU implementation.

Both implementations operated on the $1000 \times 1000 \mu \mathrm{m}$ grid, however, according to Figure 1 , in this case the crystallization occurs within the $600 \times 300 \mu \mathrm{m}$ domain. This opens room for grid optimization. The optimized grid involves $450 \times 225$ elements in the $600 \times 300 \mu \mathrm{m}$ size domain. Based on the grid dimensions, the mean grid size is $\mathrm{h}=1.33 \mu \mathrm{m}$, which is coarser than the base case grid. This is expected to generate inaccuracies, especially in the small size domain where the particle number is high due to the nucleation. To keep the small size range accurate, a non-uniform grid is applied with linearly increasing cell size, in which the size of first cell is the half of the last cell. To compare the accuracy with the base case, the standard method of moments (SMOM) is applied for the calculation of moments of the distribution: ${ }^{37}$

$$
\mu_{k, l}=\int_{0}^{\infty} L_{1}^{k} L_{m}^{l} n(\mathbf{L}, t) d \mathbf{L}
$$

Since the SMOM does not involve approximations it provides the quasi-accurate evolution of the moments. Hence, the accuracy of the HR-FVM can be estimated by comparing its moments to the SMOM. This error verification is a built-in feature of both serial and parallel CrySiV functions.

According to Table 3, the reduced non-uniform grid produces slightly less accurate solution, however, the maximum and mean percentage errors are in both cases well under $0.1 \%$ except of the $\mu_{0,1}$ moment for which it is between 0.1 and $0.15 \%$. Taking into the consideration that the 
grid optimization provided almost one order of magnitude speedup (from 11.43 to $1.79 \mathrm{~s}$ ), in overall, it has positive impact in the context of process control.

Table 3. Comparison of percentage error in the leading moments of the distribution and solution time of simulations.

\begin{tabular}{|c|c|c|c|c|}
\hline \multirow{2}{*}{ Quantity } & \multicolumn{2}{|c|}{$1000 \times 1000 \mu \mathrm{m}$ uniform grid } & \multicolumn{2}{|c|}{$600 \times 300 \mu \mathrm{m}$ non-uniform grid } \\
\hline & Mean error $[\%]$ & Max. error [\%] & Mean error $[\%]$ & Max. error [\%] \\
\hline$\mu_{0,0}$ & $1.87 \cdot 10^{-2}$ & $4.27 \cdot 10^{-2}$ & $2.91 \cdot 10^{-2}$ & $6.35 \cdot 10^{-2}$ \\
\hline$\mu_{1,0}$ & $5.40 \cdot 10^{-2}$ & $8.51 \cdot 10^{-2}$ & $5.99 \cdot 10^{-2}$ & $9.19 \cdot 10^{-2}$ \\
\hline$\mu_{0,1}$ & $1.11 \cdot 10^{-1}$ & $1.45 \cdot 10^{-1}$ & $1.14 \cdot 10^{-1}$ & $1.47 \cdot 10^{-1}$ \\
\hline$\mu_{1,2}$ & $3.06 \cdot 10^{-2}$ & $1.02 \cdot 10^{-1}$ & $2.93 \cdot 10^{-2}$ & $1.05 \cdot 10^{-1}$ \\
\hline Calculation time & $11.43 \mathrm{~s}$ & & $1.79 \mathrm{~s}$ & \\
\hline
\end{tabular}

It is known that the GPUs have better single than double precision performance for two reasons:

- executing single precision calculations is a less expensive operation than its double precision counterpart,

- depending on GPU manufacturing architecture, limited number of cores are capable to execute double precision operations.

In this simulation the type casting from double to single precision delivered simulation time reduction from 1.79 to $1.27 \mathrm{~s}$. However, this leads to inherent accuracy degradation: the typecasting error in the crystal number simulation was $1.0810^{-5} \%$. This can be considered negligible for the purpose of the work. 
Table 4. Actions taken to improve the simulation time of the 2D HR-FVM and their impact on the run time. See Appendix 1 for machine specifications.

\begin{tabular}{llccc}
\hline \multirow{2}{*}{ No. } & Action taken & Simulation & \multicolumn{2}{c}{ Speedup [-] } \\
\cline { 3 - 5 } & & time [s] & Partial & Total \\
\hline 1 & Base case: serial C implementation & 132.20 & 11.57 & 11.57 \\
2 & Hybrid CPU-GPU implementation & 11.43 & 6.39 & 73.8 \\
3 & Grid optimization, variable grid & 1.79 & 1.41 & 104.1 \\
4 & Type cast to single precision & 1.27 & & \\
\hline
\end{tabular}

According to Table 4, two orders of magnitude speedup was achieved from the base case to the final, optimized solution, which involves variable grid, single precision floating-point operations and hybrid CPU-GPU implementation. The most significant speedup comes from the use of GPU but the grid optimization was also highly beneficial, which is process-dependent. The improved simulation time enables the use of accurate, full 2D PBE simulations for real time control applications.

\section{Soft-sensor development for PVM and FBRM}

The measured data have a key role in the design of any feedback control system. For NMPC applications the measured data need to be comparable with the calculated quantities. This raises difficulties for the model based shape control, because the simulated 2D CSD currently cannot be measured with in-situ real-time tools. FBRM and PVM are the most commonly used PAT tools for crystallization monitoring, which provide the CLD and ARD, quantities, which however differ substantially from the 2D CSD. 


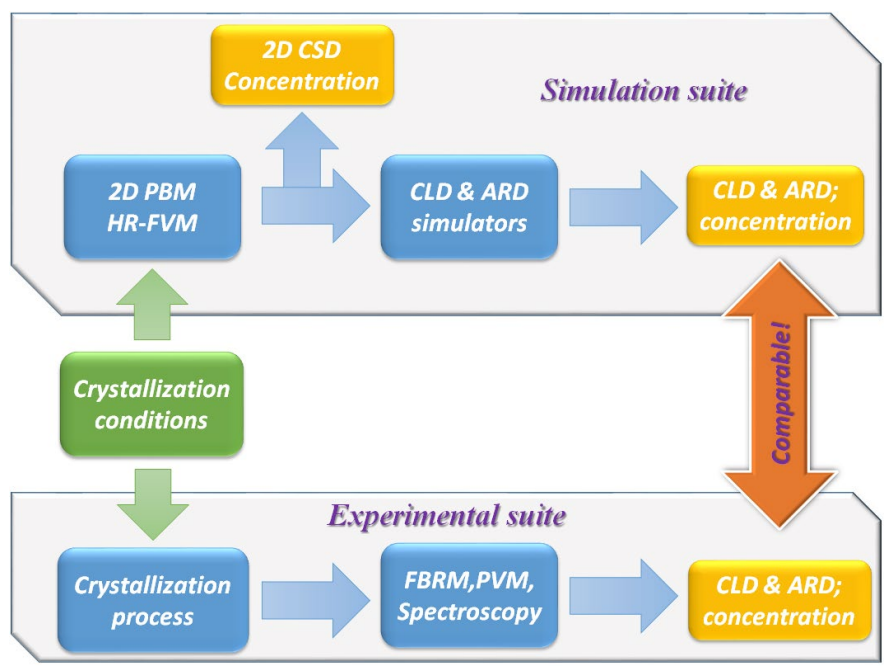

Figure 3. Schematic representation of the model structure, which enables the direct comparison of simulated data with the in-situ available measurements.

For the development of the sensor models two mathematical functions/models need to be derived with forms:

$$
\begin{aligned}
& C L D_{\mathbf{L}}=f(\mathbf{L}) \\
& A R D_{\mathbf{L}}=g(\mathbf{L})
\end{aligned}
$$

In Eq. (12) $C L D_{\mathbf{L}}$ and $A R D_{\mathbf{L}}$ denotes the CLD and ARD vectors of crystals of size $\mathbf{L}$. It would be difficult to regress some analytical functions of $f(\mathbf{L})$ and $g(\mathbf{L})$. Instead, in this work ANNs are employed. ANNs are computational systems, inspired by the biological neural networks, which found most use in applications that are difficult to express mathematically. The building block of ANN is the artificial neuron (AN), which has two interior poles (see Figure 4a). The left hand side of AN computes the weighted sum of inputs $\left(x_{i}\right)$, where an input can be an external input or the output of another neuron. The weighting factor $\left(w_{i}\right)$ adjusts the relative importance of inputs. There is a bias $(b)$, which provides an offset to the weighted sum. The right hand side of the AN calculates the output for the weighted sum, based on the activation function. In the ANN the ANs are 
connected to each other, typically in unidirectional topology: each neuron receives the inputs from a left hand side layer and are connected to a right hand side layer, as Figure $4 \mathrm{~b}$ illustrates. The layers of neurons between the inputs and outputs are the hidden layers. ${ }^{38}$

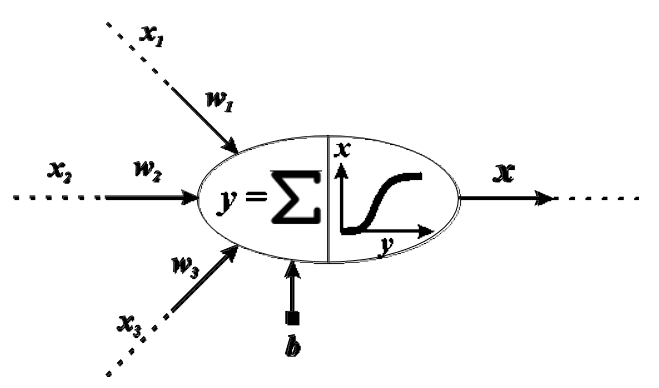

a)

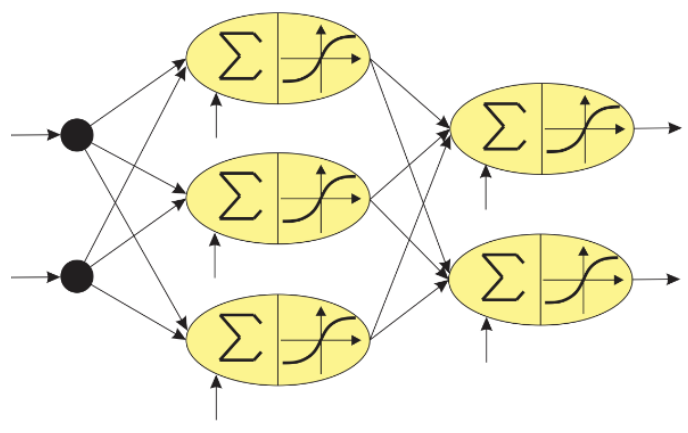

b)

Figure 4. a) Structural representation of an artificial neuron with three inputs and sigmoid transfer function; b) Illustration of the topology of a feed-forward neural network with two inputs and two outputs.

There are still debates on topology optimization of ANNs i.e. how many layers and neurons in each layer should be used for specific problems. For non-linear, i.e. sigmoidal neurons adding more layers increases the network's abstraction level. These are often referred to as deep NN (DNN). ${ }^{39}$ The training of NNs consists of the optimization of weights and biases so the calculated outputs match the output set of the training data. 
The ANN can be trained based on any other type of training data, including experimentally measured CLDs and ARDs or any model available in the literature, including different geometrical $^{40}$ or optical ${ }^{25}$ models. In this work, for the illustration of ANN training and performance, an improved geometrical model is applied. ${ }^{28}$ Although ANNs were applied to correlate the CLD to product properties ${ }^{41}$, this is the first application to simulate directly the output of the PAT tools.

\section{FBRM and PVM models}

The FBRM and PVM models presented here are not aimed to provide an accurate static conversion between CSD and CLD/ARD. These models are approximate, but also rely on the autocorrective feature provided by the dynamic feedback and the adaptive design of the controller. The transformation has two steps:

- Since the probes detect the $2 \mathrm{~d}$ projections (let " $d$ " denote the space dimensions, which should not be confused with the " $D$ " crystal dimension) of the crystals in the first step we also take a particle with size $\mathbf{L}$ and map the possible $2 \mathrm{~d}$ projections. For these projections an ARD can be constructed, which reflects the measurable ARD of the corresponding homogeneous crystal population. For the approximation of CLD, all "cuts" of all $2 \mathrm{~d}$ projections are mapped. The CL is associated with the cut length, thus approximate CLD can be constructed for the crystal of size $\mathbf{L}$. It must be noted that this step assumes random spatial orientation of particles in the slurry. For highly non-isotropic crystals, such as high aspect ratio particles, this assumption might not hold since the crystals might follow, for certain degree, the flow-field. This phenomenon is expected to depend on the properties of crystallization process (liquid and solid density), operating conditions (stirring energy, 
turbulence conditions) and crystal size and shape. The detailed modelling of these effects is far beyond the scope of the current work hence these were neglected.

- In the second step, the CLD and ARD of the crystal population given by the $2 \mathrm{D}$ CSD are approximated as the number weighted sum of the CLDs and ARDs of individual crystals. This step can be carried out in real time in lack of the ANN system ${ }^{28}$, using the 2D CSD calculated by the PBM simulation.

Figure 5 illustrates the working principle of the geometrical model based soft-sensor design: position (1) represents a random crystal orientation in the crystallizer. Position (2) is the two dimensional projection of position (1), detectable by the sensor, which depends on the crystal orientation, therefore, every crystal orientation leads to different silhouette. Position (3) is the rectangular approximation of the silhouette (Position 2), which eases considerably the automatic calculation of the CLs, and so the CLD construction. For the same random orientation, the AR $\left(L_{1, o b s} / L_{2, o b s}\right)$, which would be detected by the PVM, can also be easily calculated.

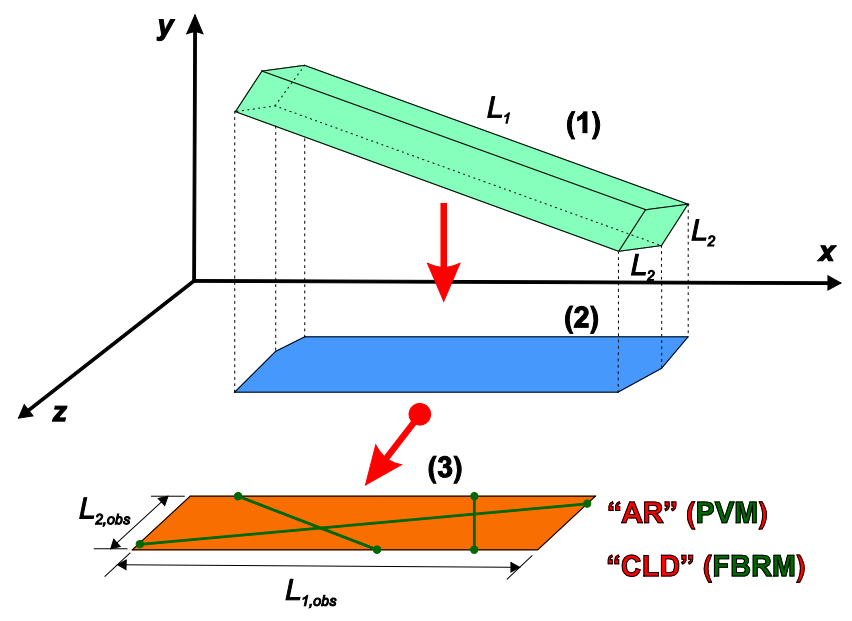

Figure 5. Working principle of CLD and ARD generation of individual high aspect-ratio crystals based on geometrical modeling by mapping all possible $2 \mathrm{~d}$ projections of the $3 \mathrm{~d}$ crystal body. 
The method has already been validated for spherical particles in the literature. ${ }^{28}$

CLDs and ARDs of individual crystals are pre-calculated for $1000 \times 1000 \mu \mathrm{m}$ grid in $\mathrm{h}=1 \mu \mathrm{m}$ discrete size increments, thus the direct transformation would be applicable on this grid only. This $10^{6}$ data sets will serve as training data for the ANN.

Figure 6 presents the upper view of the product CSD from Figure 1 as well as the corresponding simulated CLD and ARD. It is known that the high AR crystals tend to form bimodal CLD, ${ }^{42}$ which can be recognized in the simulation results. Additionally, the CLD peaks correlate well with the approximate size pair of the grown seed crystals. The third peak in the fine CL domain reflects the fines. In the ARD plot it seems that the AR maximum is lower than the expected mean AR of the population. This effect is explained with the random spatial orientation of crystals and it will be discussed in detail later in this article.

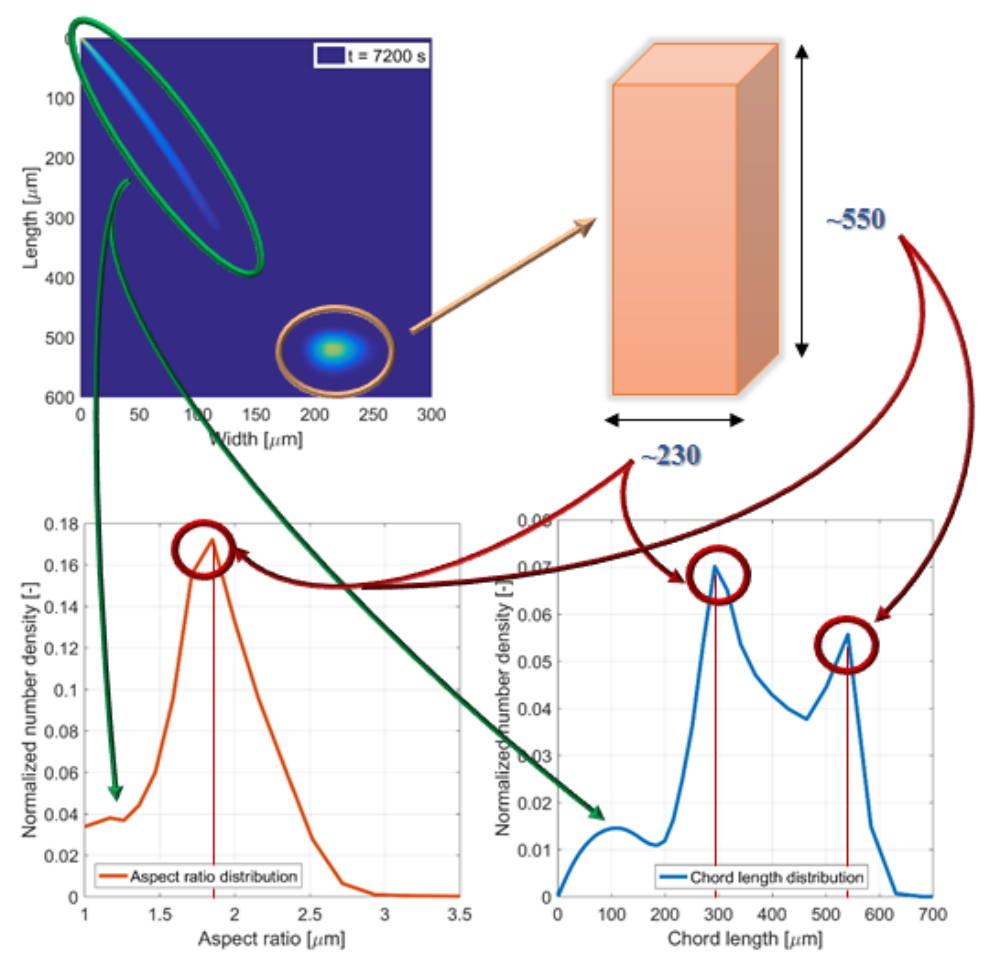


Figure 6. Simulated CLD and ARD of a typical seeded batch crystallization product with the relations between the 2D CSD and CLD and ARD.

Figure 7 depicts the temporal evolution of simulated CLD and ARD, corresponding to the $2 \mathrm{D}$ CSD variations. There is a continuous increase of CLs, due to the crystal growth, whereas the crystal shape changes only at the beginning of the process. In this simulation the CLD is normalized at $t=0$ time moment and, in order to simulate the impact of nucleation on the count, in the upcoming moments the normalized CLD is multiplied by the relative crystal number increase.
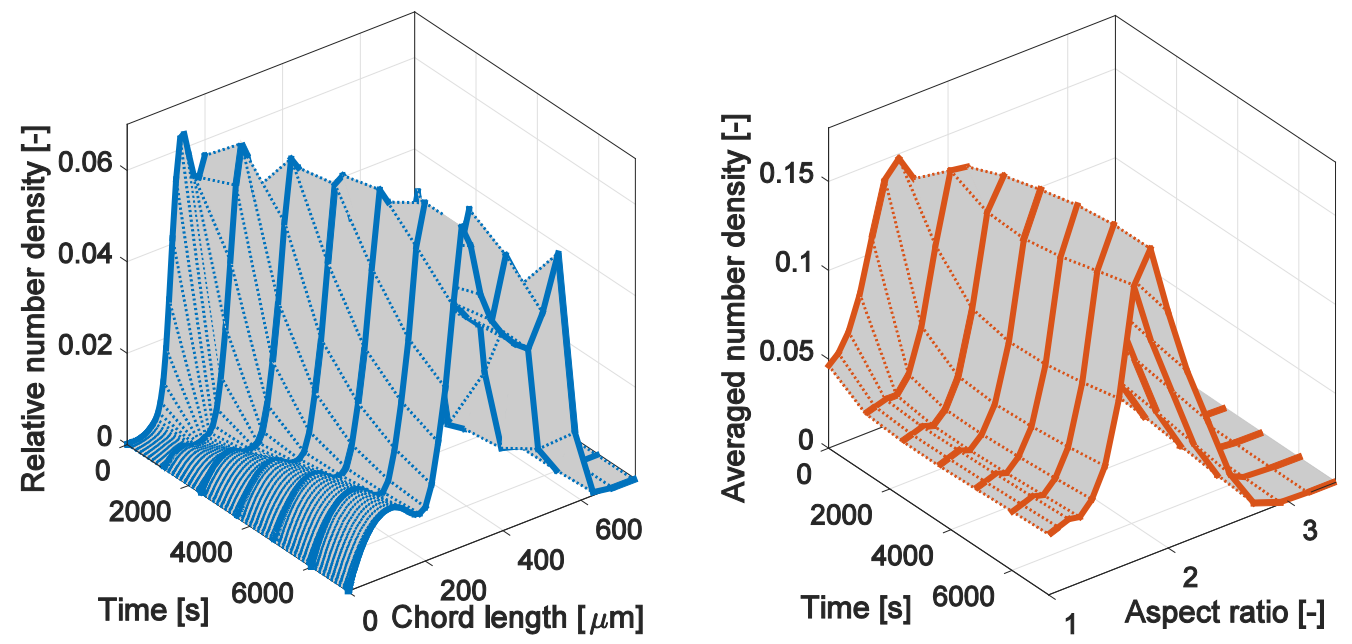

Figure 7. Simulated temporal evolution of CLD and ARD during a batch cooling crystallization.

There are seven CLD and ARD represented in Figure 7, which were generated on the full $1000 \times 10002 \mathrm{D}$ CSD grid in 6.00 seconds. Thus, one 2D CSD to CLD and ARD transformation takes 0.86 seconds. From the perspective of information technology, the operation of weighted summing is fundamentally different from the PBE solution, which involves more expensive calculations, such as power - and exponential functions, making the program more CPU intensive. 
The weighted summing is less CPU intensive, however implies more memory (RAM) access. For this reason, the programming strategies that successfully accelerated the PBM solution failed to improve the weighted sum calculation:

- The GPU implementation of CLD and ARD calculation takes $\sim 50 \%$ longer than the CPU. The explanation is that the secondary operations, such as memory allocations (GPU, RAM) and data copy from RAM to GPU, takes longer than the benefits of parallel calculations.

- The single precision serial code gave roughly the same running time as the double precision counterpart.

- Parallelizing the weighted sum calculation on the CPU leads to $\sim 10 \%$ computational time increase. This reflects that the program is optimized from the memory utilization point of view. Accessing the cache is considerably faster than the RAM. By one RAM access a sub vector is loaded into the cache. In serial mode all elements of the sub vector are processed, then a new set of sub-vector is loaded in to the cache. Therefore, the serial execution minimizes the slower RAM access.

A critical disadvantage of the described transformation is that it operates only on the precalculated $(1000 \times 1000) 2 \mathrm{D}$ CSD grid. For custom grids the new 2D CSD must be interpolated to the default 2D CSD, for which the individual CLDs and ARDs are available. Both the interpolation and calculations on an oversized grid introduce unnecessary calculations, however both of these can be avoided by using ANNs.

\section{Artificial neural network design for CLD and ARD simulation of individual crystals}

The inputs of the ANN for the prediction of CLD and ARD of arbitrary sized crystals are the crystal length and width, whereas the output is the CLD and ARD vector. For training and testing the pre-computed data on the $1000 \times 1000$ grid is used, summing up to $10^{6}$ data sets. Two separate 
networks were trained for CLD and ARD. The ANN was trained in Matlab environment using the Neural Network Toolbox. During the early development stage, it has been observed that a single or two layer ANN cannot achieve reasonable performance even with high number of neurons (up to 5000), however increasing the number of layers with considerably less neurons led to better performance. The best performance was achieved with a pyramidal topology, in which the number of neurons gradually increased from the input to the output layer. It was also observed that decreasing further the layers or the number of neurons the performance also decreased. This indicates that for this problem DNNs are required. It is worth noting that the quantity of training data points $\left(9.1 \cdot 10^{7}\right.$, as each set represents a CLD/ARD vector with 91 elements) enables the reliable optimization of the 28395 weights. The applied training function is known to provide an increased performance for networks with large number of weights. The hyperbolic tangent sigmoid function gave the fastest convergence and best performance.

Table 5. ANN training and topology related data.

\begin{tabular}{ll}
\hline Property & Value \\
\hline Network type & Feed-forward network \\
Transfer function & Hyperbolic tangent sigmoid \\
Training method & Scaled conjugate gradient backpropagation \\
Number of hidden layers & 9 \\
Number of neurons in each layer & $\{4,8,10,12,16,25,40,67,150\}$ \\
Total number of weights & 28395 \\
Number of $\{$ training, validation, & $\{800000,100000,100000\}$ \\
test $\}$ datasets
\end{tabular}


Figure 8 presents the ANN performance in test run for CLD and ARD prediction. There are a few outliers but their relative number is low, which is also supported by the $\mathrm{R}^{2}>0.997$ in both case.
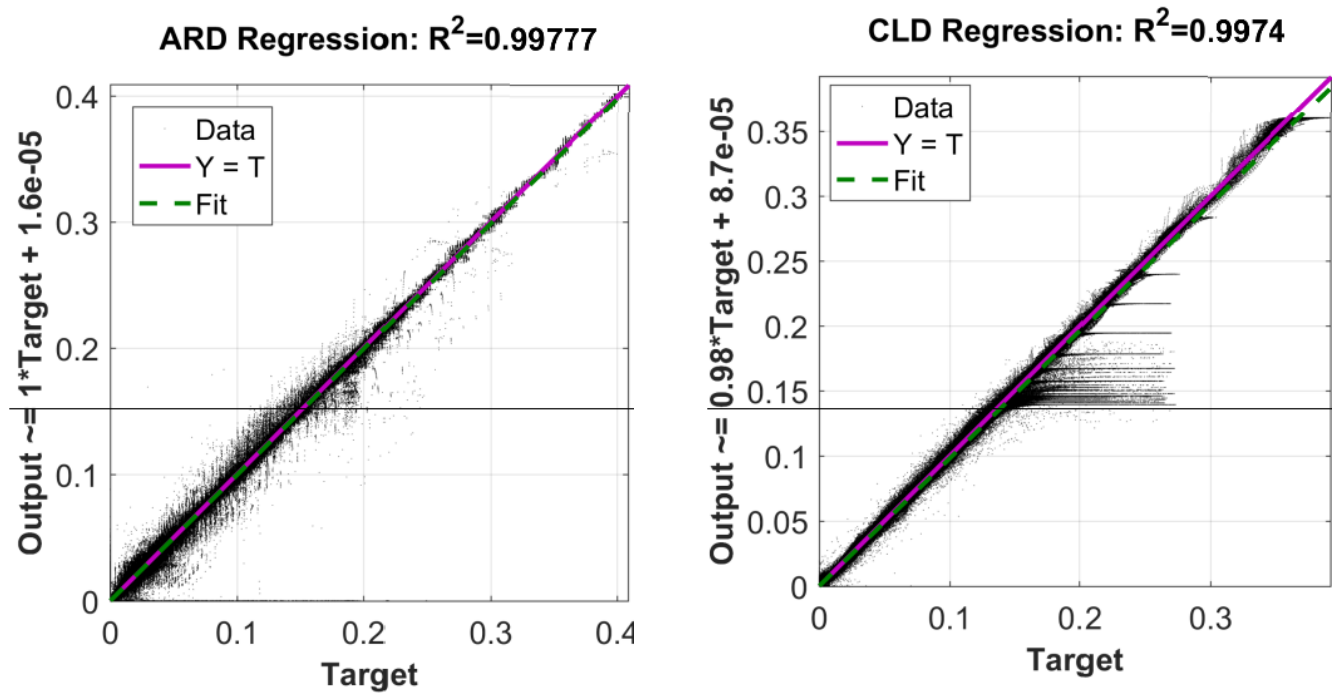

Figure 8. ANN test run performance for the prediction of the CLD and ARD.

The trained ANN is able to reproduce the CLD and ARD of arbitrary sized 2D crystals, which enables the application of 2D CSD to CLD and ARD transformation on custom 2D CSD grids.
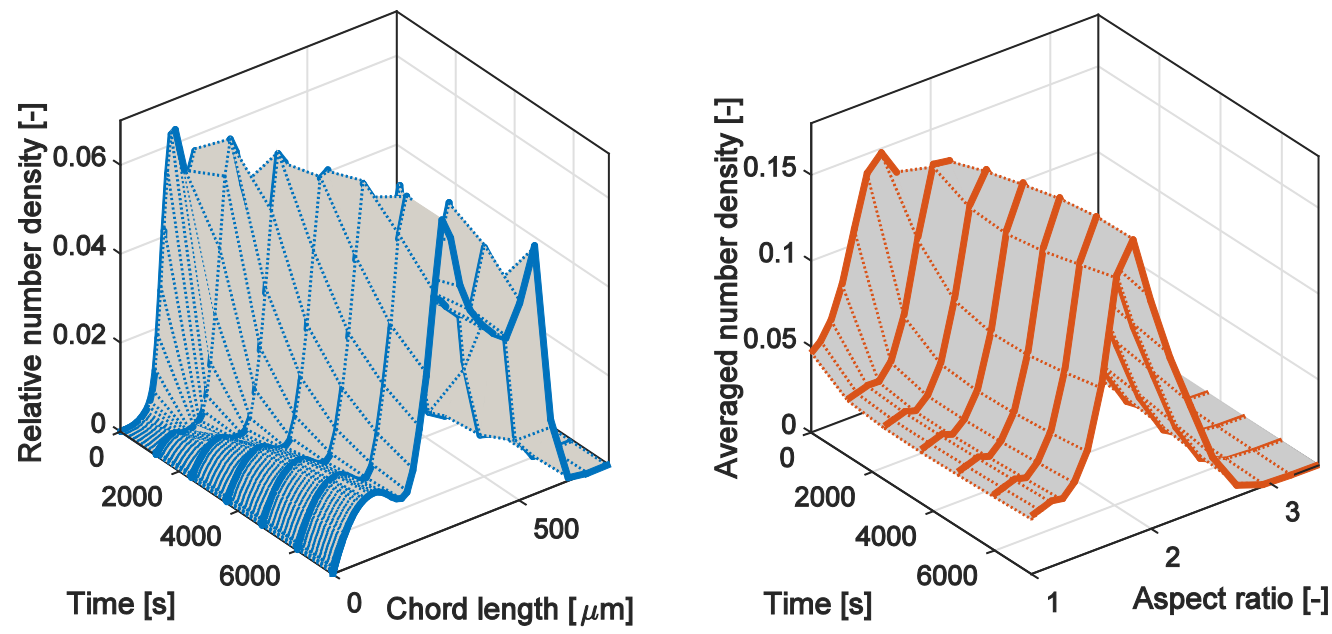

Figure 9. ANN supported simulation of CLD and ARD evolution. 
The CLD and ARD evolutions represented by the surfaces on Figure 9 were generated in 0.967 s by direct conversion on the $600 \times 300$ grid based on the data provided by the ANN. Except of the apparent noise in low size domain of the CLD surface no visible difference exists from the surfaces in Figure 6. The maximum difference between the ANN based CLD and pre-computation based CLD graph is $1.76 \cdot 10^{-3}$, whereas the mean difference is $1.63 \cdot 10^{-4}$. The same deviations for the ANN based ARD calculation are $2.21 \cdot 10^{-3}$ and $2.21 \cdot 10^{-4}$, respectively. The mean deviation is with an order of magnitude lower than the maximum deviation and with two order of magnitude lower than the maximum CLD/ARD values. This indicates that the improved calculation time of the ANN soft sensors makes them suitable for control application.

Based on the ARD graph in Figure 9 the ARDs are pre-calculated for the 1-1000 AR domain, however, the ARs are generally lower, in this case under $\sim 3.5$. To estimate the upper bound of AR calculation domain based on the 2D CSD, the mean ideal AR is calculated in terms of moments as:

$$
A R_{r}=\frac{\mu_{10}}{\mu_{01}}
$$

According to preliminary analyses the upper AR bound can be set to $1.5 A R_{r}$. With this reduction the transformation time is reduced to $0.679 \mathrm{~s}$. Table 6 summarizes the strategies and their effects on the forward transformation run time.

Table 6. Summary of the actions taken and their effects on the 2D CSD to CLD and ARD transformation time.

\begin{tabular}{lccc}
\hline \multirow{2}{*}{ No. Action taken } & Simulation & \multicolumn{2}{c}{ Speedup [-] } \\
\cline { 3 - 3 } & time $[\mathrm{s}]$ & Partial & Total \\
\hline
\end{tabular}




\begin{tabular}{lllll}
\hline 1 & Base case: $1000 \times 1000$ 2D CSD grid & 6.001 & - & - \\
2 & Variable grid (using ANN data) & 0.967 & 6.21 & 6.21 \\
3 & Reduced AR calculation domain & 0.679 & 1.42 & 8.84 \\
\hline
\end{tabular}

Finally, the 2D CSD to CLD and ARD transformation was speeded up by a factor of 8.84 , as the ANN enabled the direct transformation on the optimized non-uniform 2D CSD grid and the AR domain of transformation was delimited. The core advantage of the ANN based simulation is that it is able to keep the timing presented in Table 6 but it can be trained flexibly based on other FBRM and/or PVM models or on experimental data.

By the application of advanced computing infrastructures and ANNs, the simulation time of the $7200 \mathrm{~s}$ batch crystallization process has been reduced from $138.201(132.2+6.001) \mathrm{s}$ to 1.949 $(1.27+0.679)$ s. This represents approximately two orders of magnitude speedup, which makes the method promising for model based control applications.

\section{In-situ imaging based aspect ratio measurement: interaction with spatial orientations}

It was already demonstrated that in-situ imaging coupled with real-time image analysis are suitable measurement tools for feedback control systems. ${ }^{43}$ Moreover, these are the exclusive instruments that provide real-time shape information. The disadvantage of these systems is that the crystals are captured in random orientation (Figure 4). Therefore, it is unlikely that the real shape is measured, which is often a controlled variable. As a consequence, a correction is needed, which takes into account the offset between the real and measured AR. Let the real mean AR be defined as:

$A R_{i}=\frac{L_{1}}{L_{2}}$

Although, the observed AR of a random projection (Figure 4) can be written as: 


$$
A R_{o b s}=\frac{L_{1, o b s}}{L_{2, o b s}}
$$

Assuming rectangular silhouette of the 2D projection, the observed sizes are expressed in the function of $\alpha$ and $\beta$ angles as:

$$
\begin{aligned}
& L_{1, \text { obs }}=L_{1} \cos (\alpha)+L_{2} \sin (\alpha) \\
& L_{2, \text { obs }}=L_{2} \cos (\beta)+L_{2} \sin (\beta)
\end{aligned}
$$

Considering random crystal orientation, as in the case of the geometrical model development, e.g. $\alpha$ and $\beta$ angles are uniformly distributed random variables within the $[0, \pi / 2]$ interval that covers all possible orientations of the $2 \mathrm{D}$ crystal, the mean observed sizes are expressed by the integrals:

$$
\begin{aligned}
& \bar{L}_{1, \text { obs }}=\frac{L_{1}}{\pi / 2} \int_{0}^{\pi / 2} \cos (\alpha) d \alpha+\frac{L_{2}}{\pi / 2} \int_{0}^{\pi / 2} \sin (\alpha) d \alpha \\
& \bar{L}_{2, \text { obs }}=\frac{L_{2}}{\pi / 2}\left(\int_{0}^{\pi / 2} \cos (\beta) d \beta+\int_{0}^{\pi / 2} \sin (\beta) d \beta\right)
\end{aligned}
$$

By combining Eq.(15) and Eq.(17) the most probable captured mean AR for $\mathbf{L}$ crystal, take the form:

$$
A R_{o b s}=\frac{\bar{L}_{1, o b s}}{\bar{L}_{2, o b s}}=\frac{L_{1}+L_{2}}{2 L_{2}}=0.5+\frac{A R_{i}}{2}
$$

Eq.(18), in fact, is the correction between the real crystal shape and the in-situ observable crystal shape. The same principle is applicable for the approximation of the mean AR in the function of mean crystal sizes:

$$
\left\langle A R_{\text {Adjusted }}\right\rangle=\frac{\left\langle L_{1}\right\rangle+\left\langle L_{2}\right\rangle}{2\left\langle L_{2}\right\rangle}=0.5+\frac{\left\langle\overline{A R}_{i}\right\rangle}{2}
$$




$$
\begin{aligned}
& \left\langle L_{1}\right\rangle=\frac{\mu_{1,0}}{\mu_{0,0}} \\
& \left\langle L_{2}\right\rangle=\frac{\mu_{0,1}}{\mu_{0,0}}
\end{aligned}
$$

Figure 10 compares the mean AR evolutions calculated by the soft-sensor $\left(A R_{\text {Softsensor }}\right)$, the ideal mean $\mathrm{AR}$ ( $A R_{\text {moment based }}$ - ratio of mean length and width, that can be calculated from the moments of the distribution) and the approximate, pseudo-measured mean $\mathrm{AR}\left(A R_{\text {Adjusted }}\right)$ of the base case simulation (Figure 1). It is evident that the in-situ AR measurement significantly underestimates the real AR. By the correction Eq. (19) the $A R_{\text {Softsensor }}$ is approximated well, both in terms of absolute values and trends (increase/decline).

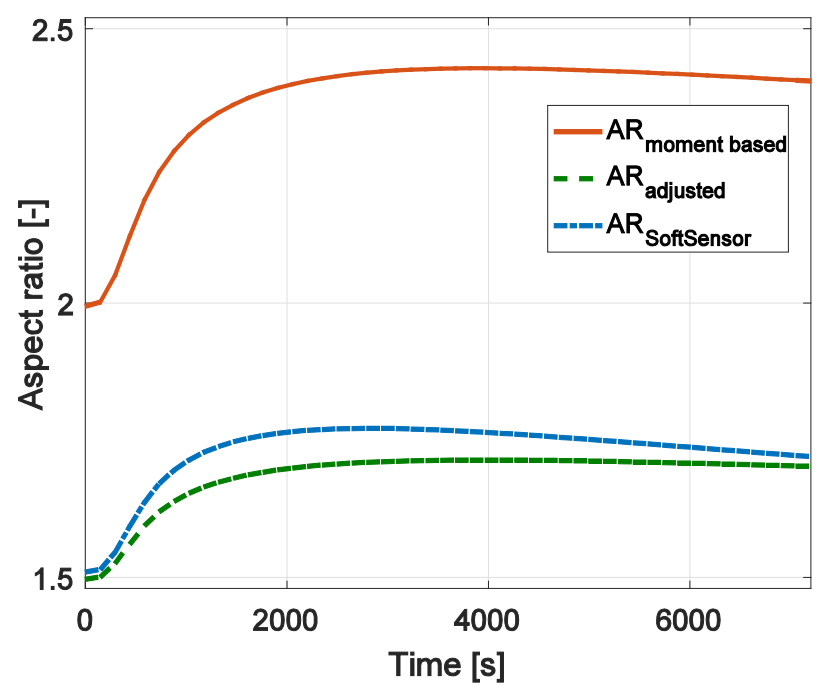

Figure 10. Random crystal orientation explains the emergence of AR underestimation in in-situ imaging based AR measurement with the proposed correction factor being able to compensate for this. 


\section{Conclusions}

In the paper a real-time applicable simulation of ARD and approximate CLD of high AR crystals during the crystallization process was presented and discussed. The HR-FVM based numerical solution of multidimensional PBEs is computationally intensive, however, in this work two order of magnitude speedup was achieved compared to the serial compiled $\mathrm{C}$ code through multiple improvements, including GPU acceleration, grid optimization and type casting to single precision floating points. These improvements enabled the real-time control application of full 2D PBEs.

An ANN based FBRM and PVM soft sensor was also developed to simulate the CLD and ARD of arbitrary sized 2D crystals. Although, the ANN can be trained based on experimental or simulated CLD and ARD data already existing in the literature, in this work an improved geometrical model was applied to calculate the most likely measurable CLD and ARD of individual 2D crystals, which was used as training and validation data for the ANN. Then, the CLD and ARD corresponding to a $2 \mathrm{D} \mathrm{CSD}$ is approximated as the number-weighted sum of individual crystal CLDs and ARDs. The ANN based simulation was faster with a factor of 6 on the optimal 2D CSD grid than the direct conversion, while the accuracy degradation was not significant.

In-situ imaging, coupled with real-time image analysis is widely used for crystallization monitoring. In this work it has been showed mathematically and through simulation that this method underestimates the real AR. For this phenomena a simple but effective correction was introduced, by which the in-situ imaging based shape information can be correlated with the real AR of crystals, enabling the direct, real shape quantitation.

\section{Corresponding Author}

*zknagy@purdue.edu, z.k.nagy@lboro.ac.uk 


\section{Acknowledgements}

The financial support of the International Fine Particle Research Institution is acknowledged gratefully. Funding from the European Research Council under the European Union's Seventh Frame-work Programme (FP7/2007-2013)/ERC grant agreement No. [280106-CrySys] is also acknowledged.

\section{Author Contributions}

The manuscript was written through contributions of all authors. All authors have given approval to the final version of the manuscript. All authors contributed equally.

\section{References}

(1) Yu, W.; Liao, L.; Bharadwaj, R.; Hancock, B. C. Powder Technol. 2017, 313, 1-8.

(2) Borsos, A.; Majumder, A.; Nagy, Z. K. Cryst. Growth Des. 2016, 16, 555-568.

(3) Larsen, P. A.; Rawlings, J. B.; Ferrier, N. J. Chem. Eng. Sci. 2006, 61, 5236-5248.

(4) Wang, X. Z.; Ma, C. Y.; Roberts, K. J. Adv. Powder Technol. 2007, 18, 707-723.

(5) Sato, K.; Nagai, H.; Hasegawa, K.; Tomori, K.; Kramer, H. J. M.; Jansens, P. J. Chem. Eng. Sci. 2008, 63, 3271-3278.

(6) Int. J. Pharm. 2017, 531, 266-275.

(7) Di Martino, P.; Beccerica, M.; Joiris, E.; Palmieri, G. F.; Gayot, A.; Martelli, S. J. Cryst. Growth 2002, 243, 345-355.

(8) Guo, M.; Fu, Q.; Wu, C.; Guo, Z.; Li, M.; Sun, J.; He, Z.; Yang, L. Colloids Surfaces B Biointerfaces 2015, 128, 410-418.

(9) Ma, C. Y.; Liu, J. J.; Wang, X. Z. Particuology 2016, 26, 1-18.

(10) Ma, C. Y.; Wang, X. Z. Chem. Eng. Sci. 2012, 70, 22-30. 
(11) Ma, C. Y.; Wang, X. Z. J. Process Control 2012, 22, 72-81.

(12) Klapwijk, A. R.; Simone, E.; Nagy, Z. K.; Wilson, C. C. Cryst. Growth Des. 2016, 16, 4349-4359.

(13) Majumder, A.; Nagy, Z. K. Chem. Eng. Sci. 2013, 101, 593-602.

(14) Reilly, A. M.; Briesen, H. J. Chem. Phys. 2012, 136.

(15) Nii, S.; Takayanagi, S. Ultrason. Sonochem. 2014, 21, 1182-1186.

(16) Wohlgemuth, K.; Schembecker, G. Comput. Chem. Eng. 2013, 52, 216-229.

(17) Ramisetty, K. A.; Rasmuson, Å. C. Cryst. Growth Des. 2018, 18, 1697-1709.

(18) Kempkes, M.; Vetter, T.; Mazzotti, M. Chem. Eng. Sci. 2010, 65, 1362-1373.

(19) Schorsch, S.; Ochsenbein, D. R.; Vetter, T.; Morari, M.; Mazzotti, M. Chem. Eng. Sci. 2014, $105,155-168$.

(20) Worlitschek, J.; Hocker, T.; Mazzotti, M. Part. Part. Syst. Charact. 2005, 22, 81-98.

(21) Fischer, C.; Peglow, M.; Tsotsas, E. Chem. Eng. Sci. 2011, 66, 2842-2852.

(22) Hukkanen, E. J.; Braatz, R. D. Sensors Actuators, B Chem. 2003, 96, 451-459.

(23) Agimelen, O. S.; Hamilton, P.; Haley, I.; Nordon, A.; Vasile, M.; Sefcik, J.; Mulholland, A. J. Chem. Eng. Sci. 2015, 123, 629-640.

(24) Kail, N.; Marquardt, W.; Briesen, H. Chem. Eng. Sci. 2009, 64, 984-1000.

(25) Kail, N.; Briesen, H.; Marquardt, W. Powder Technol. 2008, 185, 211-222.

(26) Ruf, A.; Worlitschek, J.; Mazzotti, M. Part. Part. Syst. Charact. 2000, 17, 167-179.

(27) Mesbah, A.; Nagy, Z. K.; Huesman, A. E. M.; Kramer, H. J. M.; Van Den Hof, P. M. J.; Hof, P. M. J. Van den. IEEE Trans. Control Syst. Technol. 2012, 20, 1188-1201.

(28) Szilagyi, B.; Agachi, P. S.; Nagy, Z. K. Ind. Eng. Chem. Res. 2018, 57, 3320-3332.

(29) Briesen, H. In Chem. Eng. Sci. 2006; 61, 104-112. 
(30) Oullion, M.; Puel, F.; Févotte, G.; Righini, S.; Carvin, P.; Fevotte, G.; Righini, S.; Carvin, P. Chem. Eng. Sci. 2007, 62, 833-845.

(31) Borsos, Á.; Lakatos, B. G. Chem. Eng. Res. Des. 2014, 92, 1133-1141.

(32) Gunawan, R.; Fusman, I.; Braatz, R. D. AIChE J. 2004, 50, 2738-2749.

(33) Gunawan, R.; Fusman, I.; Braatz, R. D. AIChE J. 2008, 54, 1449-1458.

(34) Majumder, A.; Kariwala, V.; Ansumali, S.; Rajendran, A. Ind. Eng. Chem. Res. 2010, 49, $3862-3872$.

(35) Szilágyi, B.; Nagy, Z. K. Comput. Chem. Eng. 2016, 91, 167-181.

(36) Ma, D. L.; Tafti, D. K.; Braatz, R. D. Ind. Eng. Chem. Res. 2002, 41, 6217-6223.

(37) Randolph, A.; Larson, M. Theory of particulate processes; Academic Press: Salt Lake City, 1973.

(38) Medler, D. a. Neural Comput. Surv. 1998, 1, 18-72.

(39) Bengio, Y. Found. Trends® Mach. Learn. 2009, 2, 1-127.

(40) Kail, N.; Briesen, H.; Marquardt, W. Part. Part. Syst. Charact. 2007, 24, 184-192.

(41) Negro, C.; Alonso, Á.; Blanco, Á.; Tijero, J. Ind. Eng. Chem. Res. 2006, 45, 197-205.

(42) Leyssens, T.; Baudry, C.; Hernandez, M. L. E. Org. Process Res. Dev. 2011, 15, 413-426.

(43) Borsos, A.; Szilagyi, B.; Agachi, P. S.; Nagy, Z. K. Org. Process Res. Dev. 2017, 21, 511 519.

\section{Synopsis}

A real time applicable approximate simulation of CLD and ARD is presented for rod-like crystals crystallization. To meet real time applicability, the HR-FVM solution of the 2D PBM equation 
with nucleation and growth is accelerated by grid optimization and GPU acceleration, whereas a fast, approximate ANN based transformation is developed for the 2D CSD to CLD and ARD transformation.

\section{Appendix}

\section{A1. Details on solution timing}

All timings presented in this work is the average of 5 runs. The machine specifications are listed in Table A1.

Table A1. The exact specifications of the machine used in timing simulations.

\begin{tabular}{ll}
\hline Property & Value \\
\hline Machine model & Dell Alienware 13 R3 \\
CPU type & Intel Core i7-7700HQ, 4 cores \\
CPU frequency (turbo) & $2.8(3.4) \mathrm{GHz}$ \\
Main memory & $16 \mathrm{~GB}$ DDR4 2667 MHz \\
GPU & nVidia GeForce GTX 1060m \\
Number of CUDA cores & 1280 \\
CUDA core frequency (turbo) & 1404 (1670) MHz \\
GPU memory (Speed) & 6 GB (8 Gbps) \\
Storage & 512 GB SSD \\
Operating system & Windows 10 professional, 64 bit \\
C compiler & Visual studio 2012 professional \\
CUDA compiler & CUDA Toolkit 8 \\
\hline
\end{tabular}


For table of content use only

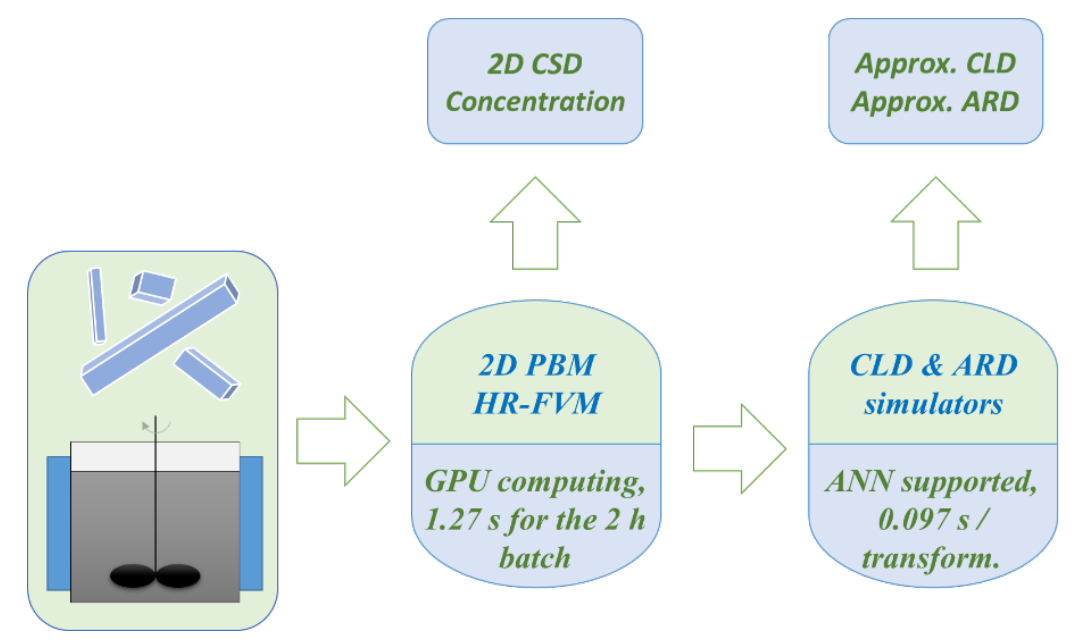

